\title{
BMJ Global Health Geospatial analysis for reproductive, maternal, newborn, child and adolescent health: gaps and opportunities
}

Zoe Matthews, ${ }^{1}$ Barbara Rawlins, ${ }^{\circ 2}$ Jennifer Duong, ${ }^{3}$ Yordanos B Molla, ${ }^{\oplus 4}$ Allisyn C Moran, ${ }^{5}$ Kavita Singh, ${ }^{6}$ Florina Serbanescu, ${ }^{7}$ Andrew J Tatem, ${ }^{8,9}$ Kristine Nilsen ${ }^{9}$

To cite: Matthews Z, Rawlins B, Duong J, et al. Geospatial analysis for reproductive, maternal, newborn, child and adolescent health: gaps and opportunities. BMJ Global Health 2019;4:e001702. doi:10.1136/ bmjgh-2019-001702

Received 7 May 2019 Revised 14 May 2019 Accepted 18 May 2019
Check for updates

(c) Author(s) (or their employer(s)) 2019. Re-use permitted under CC BY. Published by BMJ.

For numbered affiliations see end of article.

Correspondence to Professor Zoe Matthews; Zoe.Matthews@soton.ac.uk

\section{INTRODUCTION}

Reproductive, maternal, newborn, child and adolescent health (RMNCAH) indicators, such as the maternal mortality ratio, often serve as a litmus test for health system performance, because women's and children's health lies at the core of any health system. ${ }^{1}$ The health and survival of women and children does not depend on a single intervention, but on packages of interventions delivered at all levels of the health system. Mapping and tracking RMNCAH therefore captures changes in wider health system performance. But mapping has traditionally been the domain of disease-specific tracking, providing useful, but limited snapshots of progress embedded in vertical intervention mechanisms. Although disease-specific spatial mapping and research are effective ways to identify geographic inequities and to inform service provision, geographic and spatial analyses of RMNCAH have the potential to provide a broader perspective. But such analyses, especially for routine RMNCAH care provision, have been underused despite their potential to inform programmes and policies in low/middle-income countries. This commentary also argues that visualisation of RMNCAH data provides a potent social accountability and decision-making tool. Given the topic's importance, a supplement on the use of geographic information systems (GIS) in RMNCAH is long overdue.

Most geospatial studies in RMNCAH start with the geographical placement of facilities and facility staff-the fundamental infrastructure of all health systems-and the location of communities. Clearly, tackling frequently occurring birth and childhood health emergencies requires accessible, person-centred systems located near the client. ${ }^{2}$ Previous geospatial studies that have focused on determining access or distance to facilities have benefited from analytical techniques developed in the environmental science and physical geography fields. Few previous studies consider realistic travel over terrain or health facility 'bypassing,' where healthcare-seeking clients choose not to use their closest facility. This supplement thus breaks new ground by demonstrating that geospatial analysis has the potential to consider multiple important factors when assessing access to services including socioeconomic, financial, geographical and empowerment-related factors. ${ }^{3}$ Also important are measures of travel time to preferred facility and time to referral to higher level facilities. ${ }^{4}$

A recent literature review suggests there has been an increase in the number of studies exploring RMNCAH within an explicitly spatial context. ${ }^{3}$ GIS is also increasingly used to examine the relationship between maternal and child outcomes and the sociocultural environment, ${ }^{5}$ widening applicability in maternal and child health research. Spatial analysis can further uncover subnational-level differences in service coverage and health outcomes, ${ }^{6}$ which are often masked in aggregated national-level data sets. Mapping can be an important tool when used at the national and subnational levels for health service management and service planning. ${ }^{7}$

\section{AIMING FOR UTOPIA}

The papers in this supplement represent a much-needed step forward in GIS-based science for RMNCAH. However, to fully 
exploit the data and methods revolution that has been catalysed by the availability of new open-source tools for geospatial analysis, which are now accessible to low-resource settings, we need a vision for a GIS 'utopia' in RMNCAH. This commentary asks, 'What would this utopia look like?' As table 1 illustrates, we need progress on many fronts, from better data to better analytics along the impact chain from inputs to impact.

Improvements are needed across the inputs, processes/ quality of care outputs and outcomes/impact spectrum, and across UN Global Strategy domains-for example, GIS for humanitarian disasters, determinants of health for intersectoral use and analyses related to adolescent health. ${ }^{8}$ However, a GIS utopia demands more than progress. By the end of the Sustainable Development Goals (SDG) era, we envision ministries of health, non-governmental organisations and universities in all countries regularly using GIS applications to create maps that display routine and periodic health data at national and subnational levels that will aid in evidence-based planning strategies. We aim for nothing less than the routine use of such maps to inform policy development and programme management decisions, ultimately helping to reduce inequities in health service access, intervention coverage and health outcomes. These maps and other health information, such as data captured in national and district RMNCAH scorecards, will be made publicly available to foster transparency and accountability among stakeholders for progress towards national and global health goals.

Illuminating inequity is a key part of our vision for GIS in RMNCAH, but more is possible. Presenting information in a visually engaging and easy-to-understand format can facilitate data-driven decisions. As the world becomes increasingly paperless and the hunger for real-time data for strategy and accountability escalates, the use of data visualisation to organise and share information has grown. GIS analyses and tools help to present data visually so that they can be more readily comprehended by diverse audiences. However, capacity to understand and act on the data may still need to be strengthened in some settings.

\section{SEIZING OPPORTUNITIES}

Due in part to worldwide initiatives, such as the SDGs, which encourage evidence-based development, quality spatial and geographic data are increasingly available in low/middle-income countries. This development presents opportunities to take a geographic perspective for planning and strategic decision-making in global health initiatives and in RMNCAH. These opportunities involve riding the wave of emerging data availability and data analytics tools as well as engaging with large and small-scale initiatives, such as the Health Data Collaborative, which is an effort launched in 2015 by multiple global health partners to work with countries to improve the availability, quality and use of data for local decision-making and tracking progress towards the health-related SDGs.

Periodic data sources remain key to progress. As data collection moves from paper forms to mobile digital tools that can capture GIS coordinates, and health facility assessments and household surveys routinely incorporate geolocators, new analyses will be possible, including linking household and survey data. Mapping of health service statistics (national health management information system and logistics management information system data) and health facility assessment data by district presents new opportunities to examine spatial patterns in the quality of RMNCAH care. This mapping could potentially assist ministries of health to better target scarce resources for supportive supervision/mentoring and in-service training of health workers.

Data collection and analytics are also seeing more sophisticated usage as more open-source, user-friendly tools, such as Open Data Kit (https://opendatakit.org/) and QGIS (https://qgis.org), become available. Concurrently, ministries of health in low/middle-income countries are adopting electronic information systems, which has helped to increase the availability of routine health management information system data (District Health Information System 2, among the most widely used of these systems, even includes basic mapping functions).

One of the most important public health lessons from recent years is that timely information, including spatial information, is key to averting emerging disease threats, such as Zika and Ebola, and addressing humanitarian situations. Having health-related geographic information is central to creating resilient health systems. Wide acknowledgment of this reality can help us garner resources to scale up GIS capability.

\section{ADDRESSING THE GAPS}

This supplement is part of the movement to seize opportunities to move towards a GIS utopia in RMNCAH. Other initiatives, such as capacity strengthening, websites that facilitate data sharing and communities of practice, are also addressing gaps in use of GIS for RMNCAH data. Table 2 lists some examples.

\section{CONCLUSIONS}

GIS data can reveal patterns and trends to support targeted interventions and resource allocation. However, at the moment, GIS-informed policies, planning and priority setting are rare, particularly in low-resource settings where many poor health outcomes occur. There is a need to expand GIS use and galvanise efforts to improve the spatial data platforms that could strengthen application of GIS methods. A clear recognition of the role of GIS in monitoring progress towards global targets provides greater understanding of how GIS data can inform decision-making. Furthermore, this recognition leads to continued improvements in the availability and quality of geocoded data, and improved local capacity to 


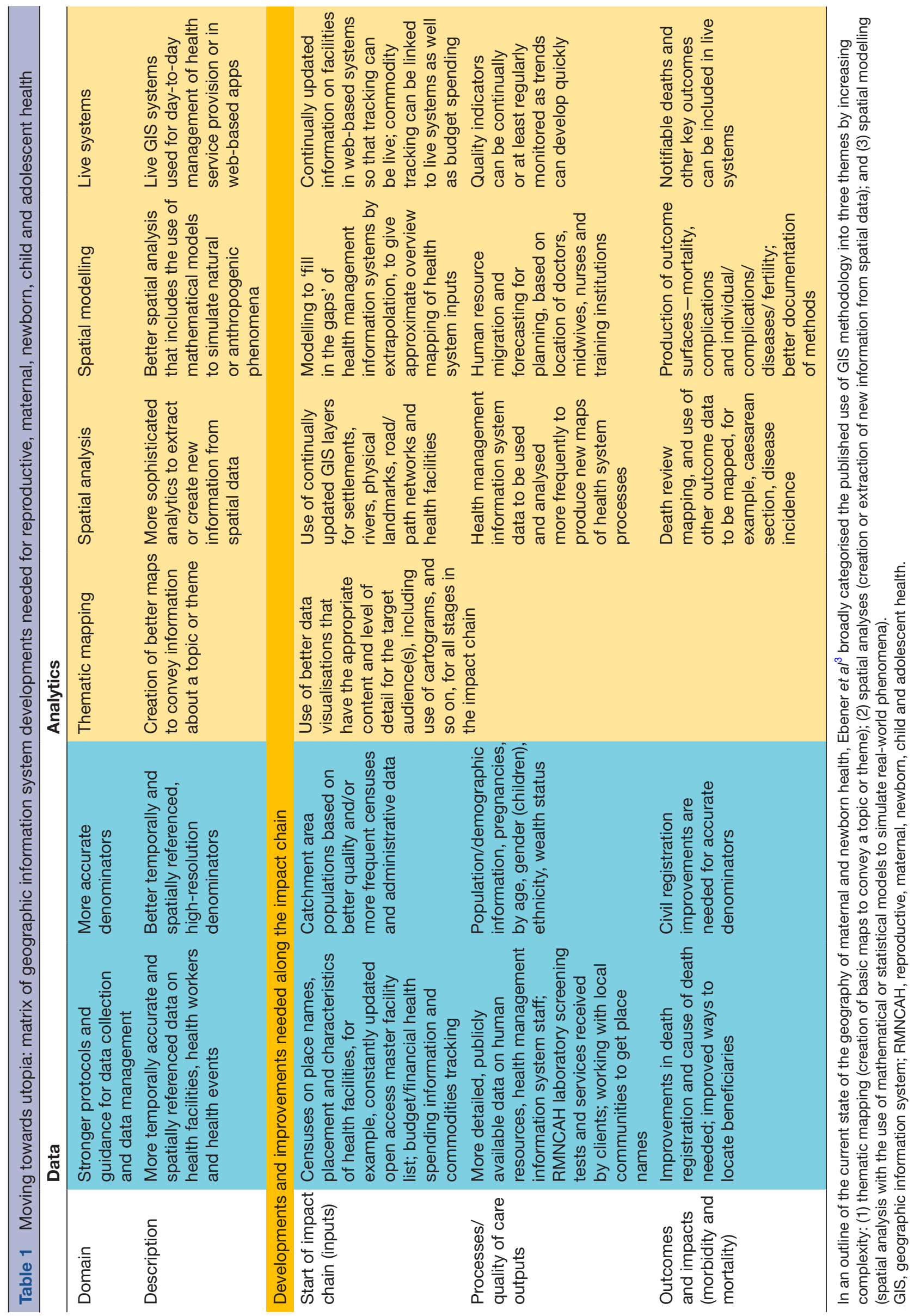




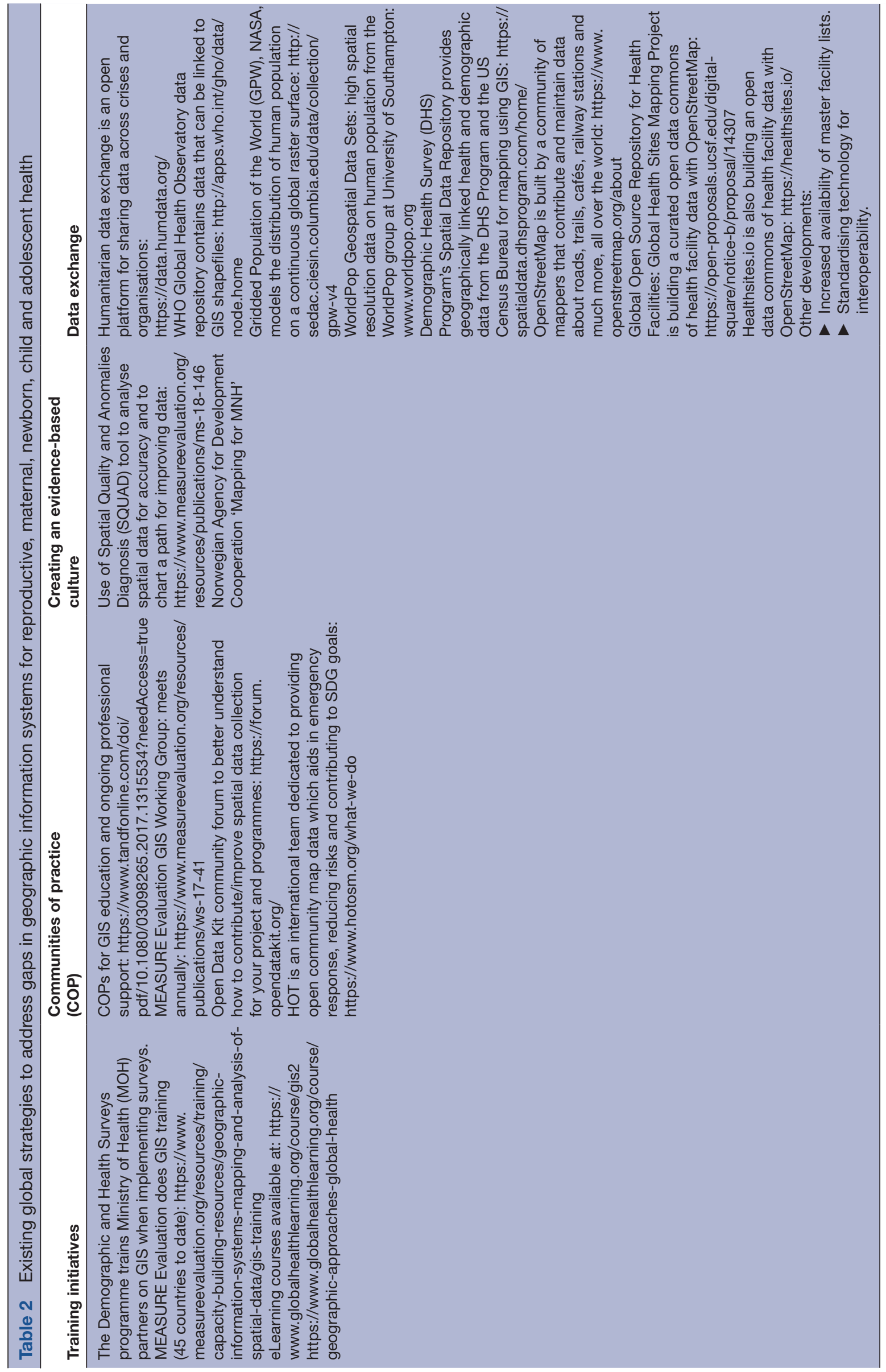


perform and interpret GIS analyses could accelerate use of these systems.

\section{Author affiliations}

${ }^{1}$ Department of Social Statistics and Demography, University of Southampton,

Southampton, UK

${ }^{2}$ MCSP, JHPIEGO, Washington, DC, USA

${ }^{3}$ GIS/IMMEL/ISD, American Red Cross, Washington, DC, USA

${ }^{4} \mathrm{MEL}$, Pathfinder International, Washington, DC, USA

${ }^{5}$ Department of Maternal, Newborn, Child and Adolescent Health, World Health Organization, Geneva, Switzerland

${ }^{6}$ MEASURE Evaluation/UNC, Chapel Hills, North Carolina, USA

${ }^{7}$ US Centers for Disease Control and Prevention, Atlanta, Georgia, USA

${ }^{8}$ Geography and Environment, University of Southampton, Southampton, UK

${ }^{9}$ WorldPop Research Group, University of Southampton, Southampton, UK

Contributors All authors contributed to the development and review of the commentary. All authors read and approved the final commentary.

Funding The manuscript is made possible by the generous support of the American people through the US Agency for International Development (USAID) under the terms of the Cooperative Agreement AID-OAA-A-14-00028.

Disclaimer The views expressed in this paper reflect the views of the authors and do not necessarily reflect those of the US Government or USAID.

Competing interests None declared.

Patient consent for publication Not required.

Provenance and peer review Not commissioned; internally peer reviewed.

Data availability statement № additional data are available.

Open access This is an open access article distributed in accordance with the Creative Commons Attribution 4.0 Unported (CC BY 4.0) license, which permits others to copy, redistribute, remix, transform and build upon this work for any purpose, provided the original work is properly cited, a link to the licence is given, and indication of whether changes were made. See: https://creativecommons.org/ licenses/by/4.0/

\section{REFERENCES}

1. Van Lerberghe W, Matthews Z, Achadi E, et al. Country experience with strengthening of health systems and deployment of midwives in countries with high maternal mortality. The Lancet 2014;384:1215-25.

2. World Health Organization. Make every mother and child count. World Health report 2005, 2005. Available: https://www.who. int/reproductivehealth/publications/maternal_perinatal_health/ 9241562900/en/

3. Ebener S, Guerra-Arias M, Campbell J, et al. The geography of maternal and newborn health: the state of the art. Int $J$ Health Geogr 2015;14.

4. Keyes E, Parker C, Zissette S, et al. Modeling geographic access to emergency obstetric services: a case study to incorporate patient bypassing and measure the impact of improvements to the emergency referral system. BMJ.

5. Makanga PT, Schuurman N, von Dadelszen P, et al. A scoping review of geographic information systems in maternal health. Int $J$ Gynaecol Obstet 2016;134:13-17.

6. Nealet al. Using GIS to support disaggregated data analysis of adolescent first births: a case study in Nepal. BMJ 2018.

7. Robinet al. Using spatial analysis and GIS to improve district level resource allocation and annual activity planning by health managers in a rural district of Bangladesh. BMJ 2018.

8. World Health Organization. Global Strategy for Women's, Children's and Adolescents' Health (2016-2030). Every Woman Every Child, 2015. Available: https://www.who.int/life-course/partners/globalstrategy/global-strategy-2016-2030/en/ 